\title{
Periosteal Osteosarcoma of Radius Mimicking Synovial Sarcoma
}

\author{
Vishnu Senthil* \\ Dept of Orthopaedics, SBMCH and Ri, India
}

Received: November 14, 2017; Published: November 30, 2017

*Corresponding author: Vishnu Senthil, Senior Resident, Dept of Orthopedics, SBMCH and Ri, Chennai, India 600044; Email: vishsnake@gmail.com

\begin{abstract}
Periosteal osteosarcoma is a rare surface osteosarcoma. It has a benign course with good prognosis. We report a case of periosteal osteosarcoma in an old lady arising around the elbow and mimicking like synovial sarcoma with calcification in the periarticular tissue. The mass biopsied proved osteosarcoma with osteoid matrix. Patient underwent definitive treatment and followed up for five years with no evidence of recurrence.
\end{abstract}

Keywords: Periosteal osteosarcoma; Calcification; Synovial sarcoma

Abbreviations: ESR: Erythrocyte Sedimentation Rate; CRP: C-Reactive Protein; MRI: Magnetic Resonance Imaging; PET: Positron emission tomography; IHC: Immuno Histo Chemistry

\section{Introduction}

Periosteal osteosarcoma belongs to the group of surface osteosarcoma. It is a rare tumour representing $1-2 \%$ of surface osteosarcoma. The tumour can mimic various benign pathological conditions when it arises around the joint and cause diagnostic dilemma. We report such a case.

\section{Case Report}

63 year old female presented with right elbow swelling which was insidious onset and progressive of 6 months duration. Dull aching pain followed swelling. Pain present at night and not relieved with analgesics. No significant loss of weight. Systemic examination appeared normal. A solitary globular swelling of dimension $5 \times 5 \times 4 \mathrm{~cm}$ present over the right elbow extending from proximal to mid forearm. Skin over the swelling was stretched with irregular surface. Swelling was firm to hard in consistency. Plane of swelling was deep to the muscle. Elbow range of motion was 50-80 degree with thumb drop and metacarpal drop. Blood investigations showed normal ESR and CRP ruling out the possibility of infection. Radiological evaluation revealed a large ill-defined lobulated lytic lesion with osteoid matrix noted within the soft tissue on volar and radial aspect of the proximal forearm. Presence of cortical destruction in the neck (metaphysis) and proximal shaft (diaphysis) of radius due to infiltration of tumour (Figure 1). MRI scan showed a large soft tissue component with mineralization and calcification. Periosteal breach with infiltration of tumour into the radial metaphysis, adjacent muscles and posterior interosseous nerve (Figure 2). Differential diagnosis of synovial sarcoma was considered. Incisional biopsy was inconclusive between low grade osteosarcoma (surface variety) and spindle cell synovial sarcoma (Figure 3). Immuno-histochemistry markers proved negative for synovial sarcoma. PET scan showed no evidence of metastasis or skip lesions. Patient counseled and opted for amputation over limb salvage. Wide local excision with above elbow amputation was performed. Tumour margins were negative and hence postoperative chemotherapy wasn't started. Now post op 5 years with no evidence of recurrence or metastasis (Figure 4).

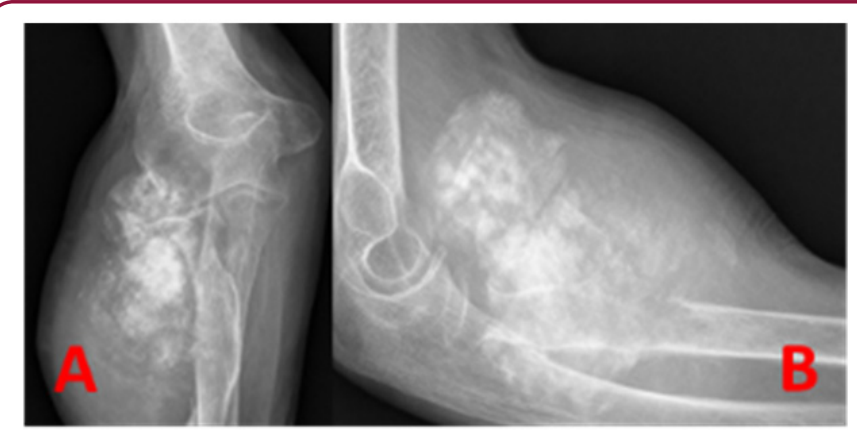

Figure 1: Radiograph of right elbow anterior-posterior(A) and lateral(B) views showing a large ill-defined roughly lobulated, lytic lesion with osteiod matrix noted within the soft tissue of volar and radial aspect of proximal right forearm. Infiltration of tumour is seen into the neck (metaphysis) of radius. 


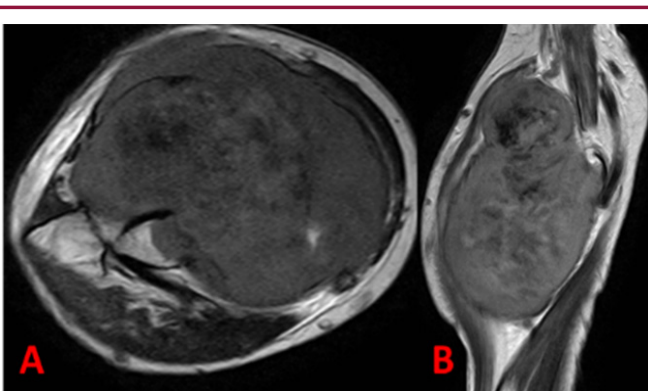

Figure 2: MRI(1.5 Tesla) of right elbow Axial T2 weighted (A) and Coronal T2 weighted (B) section showing T2 hyper-intensity in the metaphysis and in the soft tissue with cortical discontinuity. Focal T2 hypo-intensity in the soft tissue suggesting calcification.

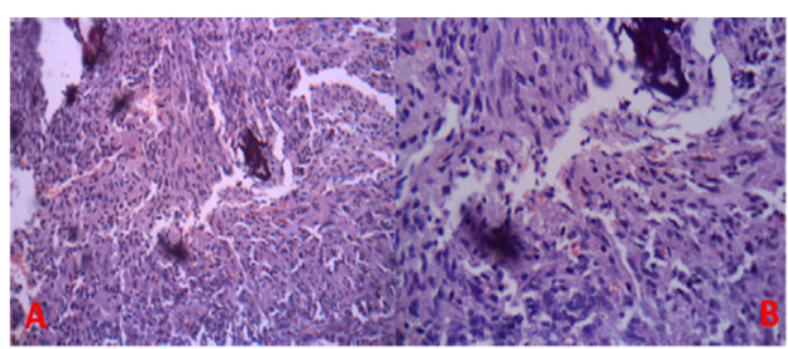

Figure 3: Microscopy slide of low power 10X (A) and High power 40X (B) showing pattern less sheets of tumour cells with ill-defined cytoplasm with moderate aniso-nucleosis. Delicate seams of osteiod with calcification are seen among the tumour cells.

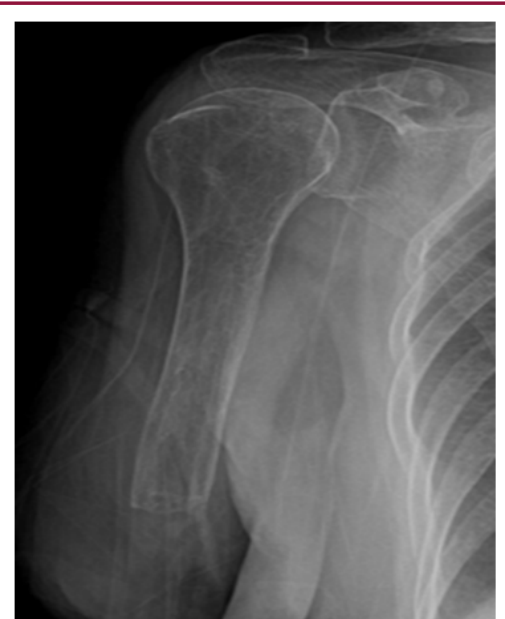

Figure 4: Anterior-posterior radiograph of the right humerus with amputation stump at follow up (5 years) showing no recurrence.

\section{Discussion}

Periosteal osteosarcoma is defined as a tumour with its epicenter outside medulla. Stippled calcification present in the tumour with or without medullary involvement [1]. Tumour commonly involves in the 3rd and 4th decade. Incidence is more common in tibia, femur and humerus. In our case, tumour is seen involving the proximal radius which is an uncommon site. Radiologically synovial sarcoma was considered as a differential because of its para-articular involvement and presence of diffuse calcification. Microscopy proved negative for synovial sarcoma because of the absence of monophasic or biphasic pattern. IHC markers were negative for cytokeratin and epithelial membrane antigen. Central medullary osteosarcoma was also excluded due to absence of a central destructive lesion with periosteal reaction. Characteristic sunburst and codman triangle with varying osteoid matrix in the soft tissue was absent [2]. Periosteal osteosarcoma has a good prognosis with high ten year survival. Primary concern of recurrence is because of positive margins and inadequate tumour resection. Lung metastasis is a problem when there is invasion of the vascular system. Presence of a meta-chronous lesion increases the frequency of metastasis. Treatment options are amputation, loco-regional control and limb salvage. Regular follow up is needed because periosteal osteosarcoma is prone for recurrence. Metachronous lesions are to be watched for, if genetic mutations are suspected [3]. Treatment for recurrence is chemotherapy and palliative radiotherapy.

\section{Conclusion}

Periosteal osteosarcoma follows a slow course and its occurrence in uncommon sites can mimic various pathological lesions. Swellings with para-articular involvement and diffuse calcification should be investigated with biopsy and IHC markers to institute prompt management. Adequate surgical clearance with chemotherapy is needed to prevent recurrence.

\section{References}

1. Kyoji Okada, Krishnan Unni K, Ronald G Swee, Franklin H Sim (1999) High grade surface Osteosarcoma, A Clinicopathological Study of 46 Cases. Cancer 85(5): 1044-1054.

2. Xin-Wei Liu, Ying Zi, Liang Bi Xiang, Tian Yu Han (2015) Periosteal Osteosarcoma: A review of Clinical Evidence. Int J ClinExp Med 8(1): 3744.

3. Matthew Revell P, Neeta Deshmukh, Robert Grimer J, Simon Carter R, Tillman RM (2002) Periosteal Osteosarcoma: a review of 17 cases with mean follow up of 52 months. Sarcoma 6: 123-130.

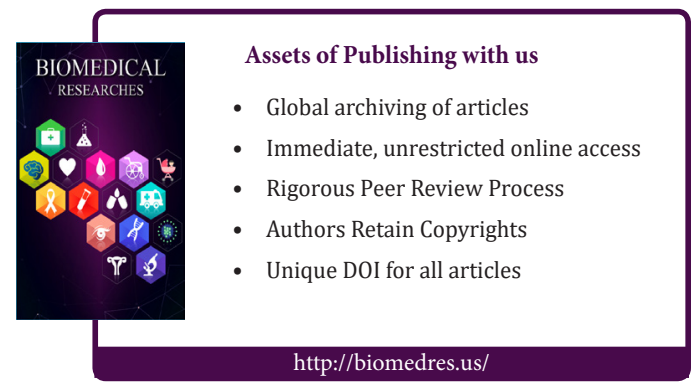

